\title{
Migraine in patients with rheumatoid arthritis and its relation to disease activity
}

\author{
Hassan Abd-Elaty El-Sonbaty ${ }^{1}$, Carmen Ali Zarad ${ }^{2}$, Mohamed Rezk Mohamed ${ }^{3}$ and Ali Ahmed Abou Elmaaty ${ }^{1 *}$
}

\begin{abstract}
Background: The comorbidity between rheumatoid arthritis (RA) and migraine is complex and not completely understood.

Objective: This study aimed to evaluate migraine frequency in patients with RA and its relation to disease activity.

Methods: A cross-sectional study was carried out on 210 consecutive RA Egyptian patients fulfilling the 2010 EULAR/ ACR criteria (joint distribution, serology, symptom duration and acute phase reaction).

Results: Prevalence of migraine in RA was 28.2\%. Disease activity, fibromyalgia and functional losses were significantly higher in migraine group with RA versus non-migraine group $(P<0.001)$. Disease Activity Score (DAS-28) was independently significant predictor as increasing DAS-28 score was associated with an increased likelihood of exhibiting migraine (5.5-times higher odds per one-unit increase in DAS-28 score). Prevalence of brain MRI white matter hyper-intensities (WMHs) in RA with migraine was 54.8\%. WMHs were significantly higher in migraine patients with aura than migraine patients without aura, especially in older patients, longer migraine duration, longer rheumatoid duration and elevated ESR $(p<0.047, p<0.034, P<0.004, P<0.015$ and $P<0.22$, respectively).

Conclusions: Migraine is highly frequent in RA patients, especially migraine with aura. The presence of rheumatoid activity, fibromyalgia and secondary Sjogren's syndrome, elevated ESR and CRP are associated with functional losses in RA patients with migraine, especially migraine with aura. MR imaging of brain is a mandatory tool for detection of white matter hyper-intensities in RA patients with migraine, especially migraine with aura.
\end{abstract}

Keywords: Migraine, Rheumatoid arthritis, Disease activity, DAS-28, MHAQ

\section{Background}

Migraine is the second most common disabling neurological disorder that commonly affects one in ten people all over the world with increased prevalence in student, women and urban residents. Migraine classified into chronic migraine $(\mathrm{CM})$ and episodic migraine (EM) depending upon the number of headache days per month $[1,2]$.

Although migraine is widely accepted disorder of central and peripheral nervous system with eminent genetic background; many previous studies reported comorbidity

\footnotetext{
*Correspondence: carmenali042@gmail.com

${ }^{1}$ Faculty of Medicine, Helwan University, Cairo, Egypt

Full list of author information is available at the end of the article
}

between systemic autoimmune disorder like rheumatoid arthritis, systemic lupus erythematosus, and thyroid dysfunction with migraine. Furthermore, presence of rheumatoid arthritis (RA) is usually associated with increased migraine pain intensity [3-5].

Rheumatoid arthritis is pro-inflammatory immunemediated disorder that symmetrically affects multijoints, especially the smaller ones. The end result of RA is progressive destruction of cartilage and bones with significant pain and wide variety of extra-articular presentations that can impair quality of life. Although the etiology of RA remains ambiguous, many factors like genetic with environmental and immunologic elements are implicated in pathogenesis $[6,7]$. 
Rheumatoid arthritis poses to be more common in migraine than in non-migraine patient. Furthermore, one recent research exhibited that migraine patients were more prone to develop rheumatoid arthritis later in life. This transitory relationship may suggest a causal link between migraine and RA $[8,9]$.

The link between migraine and RA may be clarified by presence of systemic inflammation that might increase the effect of neurogenic inflammation that present with migraine [10].

There is bidirectional mechanism between migraine and RA that can induce dysexcitability in thalamo-cortical pathway with pre-inflammatory condition in multiorgans and subsequent activation of the neuroendocrine hypothalamic and trigemino-vascular systems aiming to preserve brain homeostasis [9].

Hypothesis of development of migraine in RA patient may be attributed to tumor necrosis factor (TNF)-alpha that could produce central inflammation with subsequent central nervous system (CNS) demyelination that mediated by TNF type-1 receptor (TNFR1) leading to chronic inflammatory process with apoptosis [11].

The aim of this study was to evaluate migraine prevalence in patients with RA and its relation to disease activity.

\section{Methods}

A cross-sectional study was carried out in the period from December 2020 to June 2021 on 210 consecutive RA Egyptian patients fulfilling the 2010 EULAR/ACR criteria [12]. Patients were followed up in the Outpatient clinic of Rheumatology department. The research was approved by local Research Ethical Committee. All patients enrolled in our study provided informed written consent. The clinical and demographic data including gender, age, cigarette smoking, rheumatic arthritis disease, and duration of disease were obtained. Assessment of disease activity using Disease Activity Score in 28 joints with the erythrocyte sedimentation rate (DAS28, ESR) and current anti-RA treatment were documented. Patient functional disability was evaluated by Modified Health Assessment Questionnaire (MHAQ) [13]. We also recorded comorbidities like secondary Sjogren's syndrome, fibromyalgia and current treatments. All patients were assessed by an expert neurologist and filled a self-assessment questionnaire for migraine. We used third part of self-assessment questionnaire for migraine (Arabic version) [14]. This part of questionnaire includes questions related to time of first diagnosis, character of migraine pain (compression, pulsatile), presence or absence of aura, pain localization (unilateral, neck, bilateral), headache duration, associated symptoms (phono phobia, photophobia, nausea, vomiting), effect of activities on pain intensity and migraine attacks per month.

Exclusion criteria were any patient with autoimmune rheumatic diseases other than RA, malignancy, known renal or hepatic diseases, sepsis, critically ill, pregnancy and lactation.

Migraine diagnosis was established according to the International Classification of Headache Disorders (ICHD)-III beta criteria with stress on headache characteristics, duration, severity (assessed by Numerical Rating Scale (NRS), types (episodic or chronic), with or without aura and medications used [15].

Migraine was classified into episodic migraine (EM) and chronic migraine (CM). EM was established when patient having less than 15 headache days per month while CM defined as headache on more than or equal 15 days per month for more than or equal 3 months of which more than or equal 8 days' with criteria of migraine without aura which respond to specific medications for migraine treatment [16].

MHAQ is a self-assessment questionnaire consisting of 20 questions located in eight categories of either two or three activity of daily living [13]. DAS-28 is one of the best measures for assessment of rheumatoid arthritis disease activity. Number 28 indicates number of joints examined during assessment [14]. The results of the score were fed into a mathematical formula to produce the total Disease Activity Score and graded into: remission (DAS28 $<2.6$ ), low activity (DAS28 $\geq 2.6$ to $\leq 3.2$ ), moderate activity (DAS28 $>3.2$ to $\leq 5.1)$ and high activity $(>5.1)$. NRS requires enrolled migraine patients to rate their pain. The scale consists of the 11 point classify migraine headache into: mild (1-3 score), moderate (4-7 score) and severe ( $8-10$ score) [17].

\section{Laboratory investigations}

Complete blood count (CBC), erythrocyte sedimentation rate (ESR), $C$-reactive protein (CRP), rheumatoid factor (RF) and anti-cyclic citrullinated peptide (anti-CCP) antibody were assessed.

\section{MR imaging}

Conventional MR imaging of brain was done for all migraine patients by using 1.5-T MR machine (Siemens, Magnetom Aera, Siemens healthcare, Germany). Routine MR sequences of the brain were done including axial and sagittal T1-weighted image (repetition time $400 \mathrm{~ms}$ and echo time $14 \mathrm{~ms}$ ), axial and coronal T2-weighted image (repetition time $2500 \mathrm{~ms}$ and echo time $88 \mathrm{~ms}$ flip) and axial FLAIR images (repetition time $9000 \mathrm{~ms}$ and echo time $127 \mathrm{~ms}$ ). Diffusion-weighted imaging (DWI) and apparent diffusion coefficient (ADC) maps were done. 


\section{MR image analysis}

Brain MRI images were evaluated by an experienced radiologist with experience more than 15 years. The radiologist was blinded to the patient clinical data. Site, side and number of white matter hyper-intensities were assessed on T2WI and FLAIR images. White matter hyperintensities were visualized as small high-signal-intensity punctate foci (more than $3 \mathrm{~mm}$ ) in T2 and FLAIR images without hypo-intensity on T1-images and without diffusion restriction in DWI.

\section{Statistical analysis}

Data were coded, entered and analyzed using IBM-SPSS software (IBM Corp. Released 2017. IBM SPSS Statistics for Windows, Version 25.0. Armonk, NY: IBM Corp.). Categorical variables will be presented as $\mathrm{N}$ (\%). Chi-square test for association (Chi-square test of independence) will be used to test for the association between categorical variables. Continuous variables will be initially tested for normality using Shapiro-Wilk test and for presence of significant outliers by inspecting the boxplots. Continuous variables will be presented as means and standard deviations if normally distributed with no significant outliers; otherwise these variables will be presented as median and interquartile range, $p$ value $<0.05$ was considered statistically significant.

1. Testing for linearity: Linearity of the continuous variables with respect to the logit of the dependent variable was assessed via the Box-Tidwell procedure. A Bonferroni correction was applied using all nine terms in the model resulting in statistical significance being accepted when $p<0.0056$ (Tabachnick and Fidell) [18]. Based on this assessment, all continuous independent variables were found to be linearly related to the logit of the dependent variable.

2. Testing for outliers using case diagnostics: There was one standardized residual with a value of 2.566 standard deviations, which was kept in the analysis.

3. Testing for multi-collinearity: There was no multicollinearity as assessed by variance inflation factor $(\mathrm{VIF}=2.004,1.904,2.435$, and 1.747 for ESR, CRP, DAS28, and MHAQ, respectively).

\section{Sample size}

Sample size (n) was calculated by the following formula (Daniel and Cross) [19]:

$$
n=\frac{z^{2} \times P \times(1-P)}{d^{2}},
$$

where $Z=Z$ statistic for the level of confidence $=1.645$ for $90 \%$ confidence level, $P=$ expected prevalence $=0.274$. This prevalence was based on a previous review by Mathieu et al. [10] who reported a prevalence of migraine of $27.4 \%$ in patients with rheumatoid arthritis (RA), and $d=$ allowable (acceptable) margin of error $= \pm 5 \%$ since $P$ falls in the range of 0.1 to $0.9(d=0.05)$.

A total sample size of 215 RA patients achieves a 90\% confidence level for expected prevalence of $27.4 \%$ and an acceptable margin of error of $\pm 5 \%[10,19]$.

\section{Results}

Our results revealed that the prevalence of migraine in RA patients was $28.2 \%$.

Table (1) shows a statistically significantly higher ESR, CRP, DAS-28 score and MHAQ score in those with migraine versus those without migraine. Also higher statistically significant fibromyalgia in migraine group versus non-migraine group, secondary Sjogren's syndrome presents in $11.4 \%$ in migraine group but not present in nonmigraine group.

A binomial logistic regression was performed to ascertain the effects of ESR, CRP, DAS-28 score and MHAQ score on the likelihood that RA participants have migraine. The logistic regression model was statistically significant, $\chi^{2}(4)=63.449, p<0.001$, the model explained $36.0 \%$ (Nagelkerke R2) of the variance in migraine and correctly classified $83.2 \%$ of cases. Sensitivity was $53.2 \%$, specificity was $94.9 \%$, positive predictive value was $80.5 \%$ and negative predictive value was $83.8 \%$.

Of the four predictor variables, only DAS-28 score was statistically significantly independent predictor (as shown in Table (2). Increasing DAS-28 score was associated with an increased likelihood of exhibiting migraine (5.5-times higher odds per one-unit increase in DAS-28 score).

According to the number of white matter hyper-intensities there were 8 patients had single lesion (23.5\%) (Figs. 1, 2), 6 patients had two lesions (17.6\%) (Fig. 3) and 20 patients had more than two lesions (58.9\%) (Figs. 4, 5).

Table (3) shows a statistically significantly higher frequency of aura in those with abnormal MRI versus those with normal MRI.

Table (4) shows a statistically significantly higher ESR in those with abnormal MRI versus those with normal MRI and a statistically significantly older age, duration of RA, and duration of migraine in those with abnormal MRI versus those with normal MRI.

\section{Discussion}

The comorbidity between RA and migraine is complex and not completely understood, but many clinical researches, epidemiological and laboratory evidences support this relationship [9]. 
Table 1 Comparisons between RA patients with and without migraine

\begin{tabular}{|c|c|c|c|c|}
\hline Parameter & Non-migraine group & Migraine group & $x^{2}$ & $P$ value \\
\hline$N(\%)$ & $158(71.8 \%)$ & $62(28.2 \%)$ & & \\
\hline \multicolumn{5}{|l|}{ Qualitative data } \\
\hline Sex & & & 0.298 & 0.585 \\
\hline Male & $25(15.8 \%)$ & $8(12.9 \%)$ & & \\
\hline Female & $133(84.2 \%)$ & $54(87.1 \%)$ & & \\
\hline Current smoking & $18(11.4 \%)$ & $9(14.5 \%)$ & 0.404 & 0.525 \\
\hline Positive RF & $109(69 \%)$ & $43(69.4 \%)$ & 0.003 & 0.958 \\
\hline Positive anti-CCP & $124(78.5 \%)$ & $54(87.1 \%)$ & 2.140 & 0.144 \\
\hline Rheumatoid disease activity & & & FET & $<0.001$ \\
\hline Remission (DAS28 < 2.6) & $62(39.2 \%)$ & $6(9.7 \%)$ & & \\
\hline Low (DAS28 $\geq 2.6$ to $\leq 3.2)$ & $64(40.5 \%)$ & $17(27.4 \%)$ & & \\
\hline Moderate (DAS28 > 3.2 to $\leq 5.1)$ & $31(19.6 \%)$ & $37(59.7 \%)$ & & \\
\hline High (DAS28 > 5.1) & $1(0.6 \%)$ & $2(3.2 \%)$ & & \\
\hline Functional losses (MHAQ) & & & 21.243 & $<0.001$ \\
\hline Mild (MHAQ $<1.3)$ & $99(62.7 \%)$ & $21(33.9 \%)$ & & \\
\hline Moderate (MHAQ 1.3 to 1.8 ) & $46(29.1 \%)$ & $23(37.1 \%)$ & & \\
\hline Severe $(\mathrm{MHAQ}>1.8)$ & $13(8.2 \%)$ & $18(29 \%)$ & & \\
\hline Fibromyalgia & $1(0.6 \%)$ & $14(22.6 \%)$ & FET & $<0.001$ \\
\hline Sjogren's syndrome & $0(0 \%)$ & $7(11.4 \%)$ & FET & $<0.001$ \\
\hline NSAIDs use & $13(8.2 \%)$ & $5(8.1 \%)$ & 0.002 & 0.968 \\
\hline Corticosteroid use & $22(13.9 \%)$ & $9(14.5 \%)$ & 0.013 & 0.910 \\
\hline Methotrexate use & $114(72.2 \%)$ & $38(61.3 \%)$ & 2.460 & 0.117 \\
\hline Sulfasalazine use & $12(7.6 \%)$ & $5(8.1 \%)$ & FET & 1.000 \\
\hline Leflunomide use & $7(4.4 \%)$ & $3(4.8 \%)$ & FET & 1.000 \\
\hline Anti-TNF-a use & $4(2.5 \%)$ & $3(4.8 \%)$ & FET & 0.405 \\
\hline \multirow[t]{2}{*}{ Hydroxychloroquine use } & $77(50 \%)$ & $29(46.8 \%)$ & 0.185 & 0.667 \\
\hline & & & $Z$ value & $P$ value \\
\hline \multicolumn{5}{|l|}{ Quantitative data } \\
\hline Age (years) & $42(35-47)$ & $41(35.8-49)$ & -0.037 & 0.971 \\
\hline Rheumatoid disease duration (years) & $8(5-9.25)$ & $8(5-10)$ & -0.227 & 0.820 \\
\hline ESR & $55(49-60)$ & $64.5(50.8-79)$ & -3.766 & $<0.001$ \\
\hline CRP & $37(33-40)$ & $44(35-55)$ & -4.660 & $<0.001$ \\
\hline Disease Activity Score (DAS-28) & $2.8(2.5-3.2)$ & $3.9(2.9-4.1)$ & -6.727 & $<0.001$ \\
\hline MHAQ & $1.0(1.0-1.40)$ & $1.4(1.0-2.0)$ & -4.436 & $<0.001$ \\
\hline
\end{tabular}

Bod values indicate $P$ value is significant (less than 0.05 )

Qualitative data are N\%), test of significance is Chi-square test or Fisher's exact test (FET). $P$ value significant $<0.05$

Quantitative data are median (25th percentile-75th percentile), test of significance is Mann-Whitney U-test

RF, rheumatoid factor; Anti-CCP, anti-citrullinated peptide; DAS, Disease Activity Score; MHAQ, Modified Health Assessment Questionnaire; NSAID, non-steroidal antiinflammatory drug; TNF-a, tumor necrosis factor-alpha; ESR, erythrocyte sedimentation rate; CRP, C-reactive protein

Table 2 Predictors of the likelihood of occurrence of migraine in RA

\begin{tabular}{lllccccc}
\hline Predictor & $\boldsymbol{B}$ & S.E & Wald & Df & $\boldsymbol{P}$ & OR & 95\% Cl for OR \\
\hline ESR & -0.002 & 0.019 & 0.012 & 1 & 0.912 & 0.998 & $0.961-1.036$ \\
CRP & 0.035 & 0.027 & 1.613 & 1 & 0.204 & 1.035 & $0.981-1.092$ \\
DAS-28 & 1.707 & 0.416 & 16.862 & 1 & $<\mathbf{0 . 0 0 1}$ & 5.513 & $2.441-12.454$ \\
MHAQ & 0.059 & 0.592 & 0.010 & 1 & 0.921 & 1.061 & $0.333-3.382$ \\
\hline
\end{tabular}

Bod values indicate $P$ value is significant (less than 0.05 )

$\mathrm{OR}$, odds ratio; DAS-28, Disease Activity Score; RA, rheumatoid arthritis

MHAQ, Modified Health Assessment Questionnaire; ESR, erythrocyte sedimentation rate; CRP, C-reactive protein 


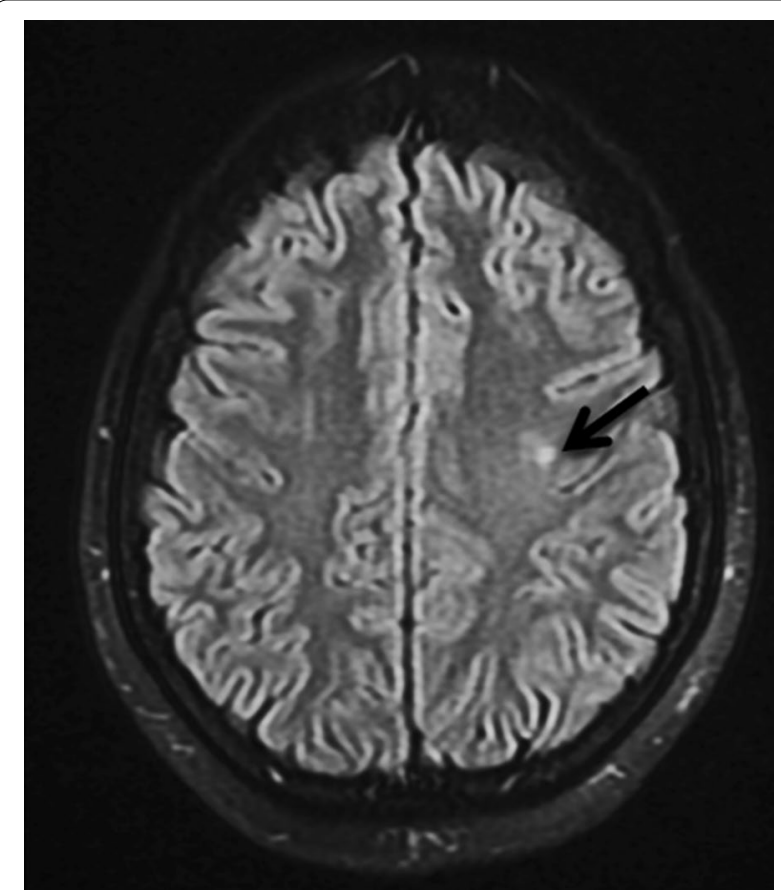

Fig. 1 Axial MRI brain FLAIR image of a 29-year-old female patient with RA and migraine with aura for 7 years showing single small white matter hyper-intensity in the left centrum semiovale (arrows)

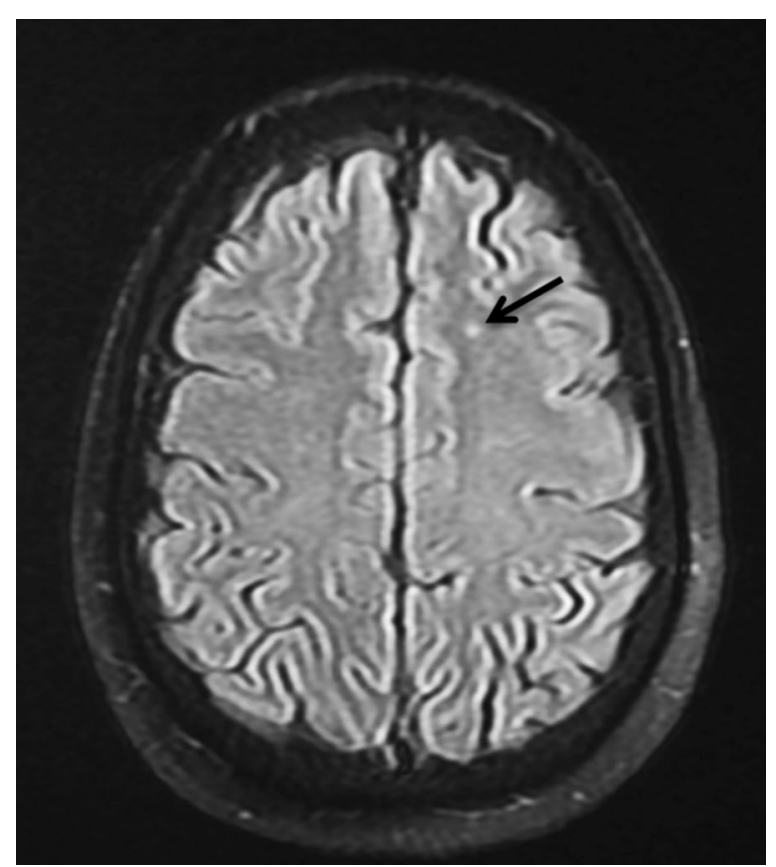

Fig. 2 Axial MRI brain FLAIR images of a 32-year-old female patient with RA and migraine with aura for 12 years showing single small white matter hyper-intensity in the centrum semiovale (black arrow)

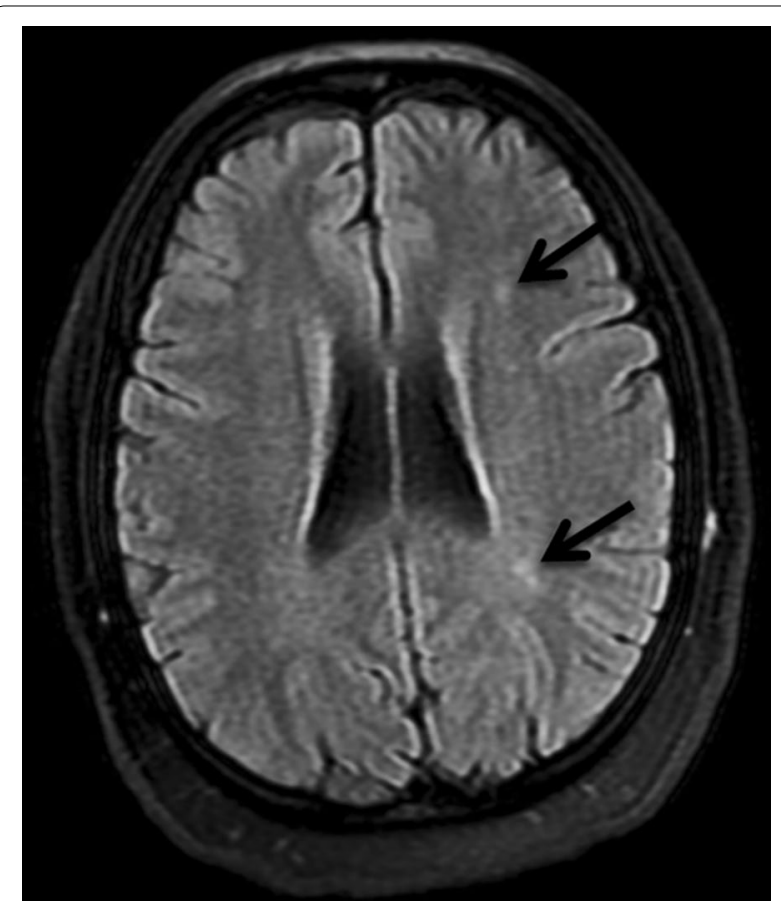

Fig. 3 Axial MRI brain FLAIR images of a 38-year-old male patient with RA and migraine with aura for 12 years showing two small white matter hyper-intensities in the periventricular white matter of left frontal and occipital lobes (black arrows)

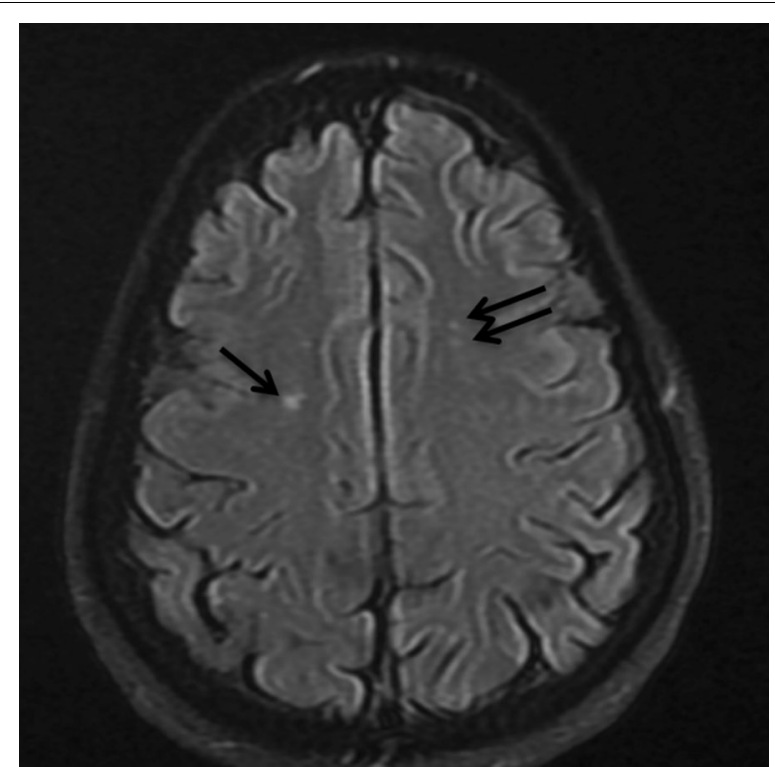

Fig. 4 Axial MRI brain FLAIR images of a 44-year-old female patient with RA and migraine with aura for 22 years showing multiple bilateral small white matter hyper-intensities in centrum semiovale (black arrows) 


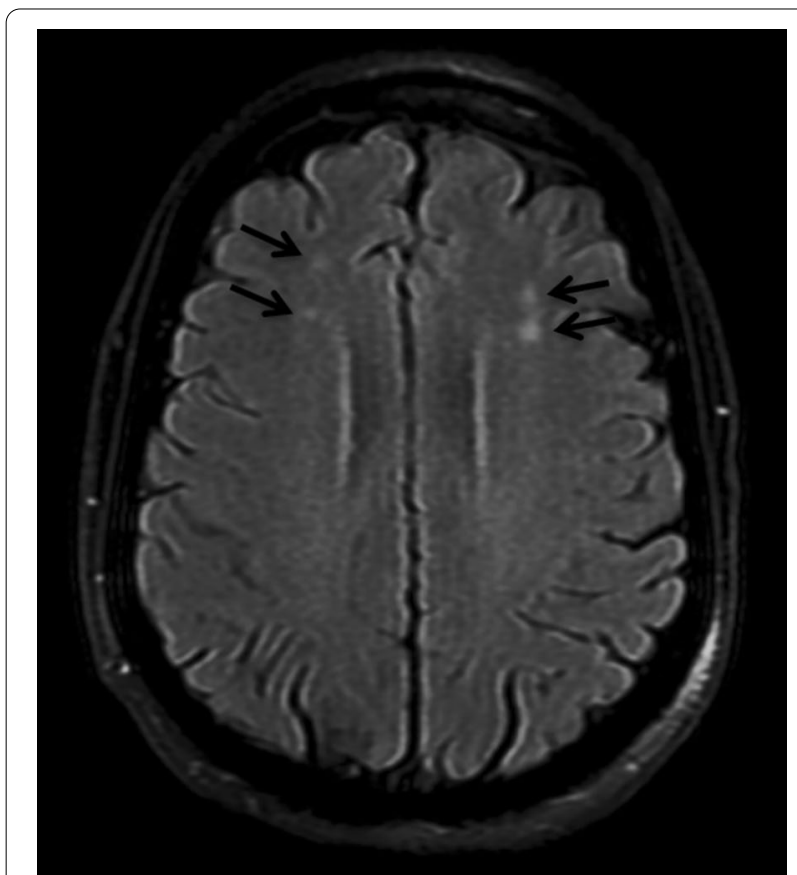

Fig. 5 Axial MRI brain FLAIR images of a 42-year-old female patient with RA and migraine with aura for 18 years showing multiple bilateral small white matter hyper-intensities in centrum semiovale (black arrows)

The comorbidity between migraine and RA has been attributed to share of serotonergic system disorder [20], significant decrease in platelet serotonin levels in RA patients and inversely related to prominent clinical rheumatoid activity [21]. The inflammatory cytokines production like TNF- $\alpha$ was inhibited by using serotonin reuptake inhibitor medication [22, 23].

In the current study, migraine prevalence was $28.2 \%$ in Egyptian random sample of 220 RA patients. Migraine was more common in females with RA, although statistically non-significant. This agrees with Mathieu and colleagues, who found that migraine was present in $27.4 \%$ of their study with female predominance [10]. Namas and colleagues reported different results in their retrospective cohort study where the prevalence of migraine in their study was as low as $5.6 \%$. This discrepancy may be due to the difference in study design, psychogenic, socioeconomic and lifestyle elements that may affect the development of migraine in RA [24].

This study revealed that there is no statistically significant difference regarding age, rheumatoid disease duration, current smoking, positive rheumatoid factor, positive anti-CCP, corticosteroid, methotrexate, sulfasalazine and anti-TNF- $\alpha$ uses between non-migraine and migraine groups with RA.
With regard to rheumatoid disease activity, our study revealed that patients in remission (DAS28 $<2.6)$ and low rheumatoid disease activity (DAS28 $\geq 2.6$ to $\leq 3.2$ ) were higher in non-migraine group of RA patients while moderate (DAS28 $>3.2$ to $\leq 5.1$ ) was higher among migraine group with RA with highly statistically significantly different in migraine group with RA $(P<0.001)$. This was in agreement with Mathieu and colleagues who found that there was statistically significant difference between migraine and nonmigraine group with RA with regard to DAS-28 [10]. As in patients with higher grades of disease activity (migraine group), there are higher levels of synovial and serum TNFalpha as TNF-alpha positively correlates with rheumatoid arthritis disease activity [24]. TNF-alpha could produce central inflammation with subsequent central nervous system (CNS) demyelination mediated by TNF type- 1 receptor (TNFR1) (a soluble form which mainly acts on the TNF type-1 receptor). TNFR1 binding leads to chronic inflammatory process with apoptosis, which is suggested to have a role in development of migraine in RA patients [25].

In our study, functional losses (MHAQ) was higher (moderate + severe $=66.12 \%$ ) in the migraine group when compared with the non-migraine group (moderate + severe $=37.34 \%$ ) with higher statistically significant difference in the migraine group $(P<0.001)$. This can be explained by the higher disease activity and the burden of migraine on quality of life and daily activities.

We found that fibromyalgia was statistically significantly higher in migraine group versus non-migraine group $(P<0.001)$. This was in agreement with Akdag Uzun and colleagues [26] and may be explained by the dopaminergic disorder but it was not in agreement with Mathieu and colleagues, who found that no significant association was present between migraine and fibromyalgia [10].

In our study, secondary Sjogren's syndrome was statistically significantly higher in migraine patients $(P<0.001)$. It was in agreement with many studies that found migraine was highly statistically significant in Sjogren's syndrome than in the control subjects and was the most common neurological complaint among these patients. This association could be a part of a common inflammatory process that occur in both conditions [27-29]. However, other researches denied this association [30].

In this research, we found that ESR, CRP, DAS-28 and MHAQ were statistically significant higher in migraine group when compared with non-migraine group $(p<0.001)$. However, the only predictors of the likelihood of occurrence of migraine in RA was DAS-28 $(p<0.001)$.

Many studies concluded that white matter hyper-intensities (WMHs) are common imaging findings in MRI brain of migraine patients [31]. The prevalence of WMH in migraine patients were varied in different studies ranging from high prevalence as in Le Pira and colleagues and 
Table 3 Comparisons of categorical data between migraine cases according to MRI findings

\begin{tabular}{|c|c|c|c|c|}
\hline Qualitative data & Normal brain MRI & Abnormal brain MRI & $x^{2}$ & $P$ value \\
\hline$N(\%)$ & $28(45.2 \%)$ & $34(54.8 \%)$ & & \\
\hline Sex & & & FET & 0.450 \\
\hline Male & $5(17.9 \%)$ & $3(8.8 \%)$ & & \\
\hline Female & $23(82.1 \%)$ & $31(91.2 \%)$ & & \\
\hline Current smoking & $3(10.7 \%)$ & $6(17.6 \%)$ & FET & 0.494 \\
\hline Positive RF & $20(71.4 \%)$ & $23(67.6 \%)$ & 0.103 & 0.748 \\
\hline Positive anti-CCP & $23(82.1 \%)$ & $31(91.2 \%)$ & FET & 0.450 \\
\hline Rheumatoid Disease Activity & & & FET & 0.393 \\
\hline Remission (DAS28 < 2.6) & $3(10.7 \%)$ & $3(8.8 \%)$ & & \\
\hline Low (DAS28 $\geq 2.6$ to $\leq 3.2$ ) & $10(35.7 \%)$ & $7(20.6 \%)$ & & \\
\hline Moderate (DAS28 $>3.2$ to $\leq 5.1)$ & $15(53.6 \%)$ & $22(64.7 \%)$ & & \\
\hline High (DAS28 > 5.1) & $0(0 \%)$ & $2(5.9 \%)$ & & \\
\hline Functional losses (MHAQ) & & & 0.754 & 0.686 \\
\hline Mild $(\mathrm{MHAQ}<1.3)$ & $11(39.3 \%)$ & $10(29.4 \%)$ & & \\
\hline Moderate (MHAQ 1.3 to 1.8) & $10(35.7 \%)$ & $13(38.2 \%)$ & & \\
\hline Severe $(\mathrm{MHAQ}>1.8)$ & $7(25 \%)$ & $11(32.4 \%)$ & & \\
\hline Fibromyalgia & $6(21.4 \%)$ & $8(23.5 \%)$ & 0.039 & 0.844 \\
\hline Sjogren's syndrome & $3(10.7 \%)$ & $4(11.8 \%)$ & FET & 1.000 \\
\hline NSAIDs use & $0(0 \%)$ & $5(14.7 \%)$ & FET & 0.058 \\
\hline Corticosteroid use & $6(21.4 \%)$ & $3(8.8 \%)$ & FET & 0.277 \\
\hline Methotrexate use & $18(64.3 \%)$ & $20(58.8 \%)$ & 0.193 & 0.660 \\
\hline Sulfasalazine use & $2(7.1 \%)$ & $3(8.8 \%)$ & FET & 1.000 \\
\hline Leflunomide use & $1(3.6 \%)$ & $2(5.9 \%)$ & FET & 1.000 \\
\hline Anti-TNF-a use & $0(0 \%)$ & $3(8.8 \%)$ & FET & 0.245 \\
\hline Hydroxychloroquine use & $13(46.4 \%)$ & $16(47.1 \%)$ & 0.002 & 0.961 \\
\hline Migraine type & & & FET & 1.000 \\
\hline Episodic & $25(89.3 \%)$ & $30(88.2 \%)$ & & \\
\hline Chronic & $3(10.7 \%)$ & $4(11.8 \%)$ & & \\
\hline Migraine with aura & $5(17.9 \%)$ & $14(41.2 \%)$ & 3.929 & 0.047 \\
\hline Numerical Rating Scale & & & FET & 0.445 \\
\hline Mild & $7(25 \%)$ & $4(11.8 \%)$ & & \\
\hline Moderate & $9(32.1 \%)$ & $12(35.3 \%)$ & & \\
\hline Severe & $12(42.9 \%)$ & $18(52.9 \%)$ & & \\
\hline
\end{tabular}

Bod values indicate $P$ value is significant (less than 0.05 )

Data are N\%), test of significance is Chi-square test or Fisher's exact test (FET). $p$ value significant $<0.05$. Quantitative data are median (25th percentile-75th percentile), test of significance is Mann-Whitney U-test. MRI, magnetic resonance imaging; RF, rheumatoid factor; Anti-CCP, anti-citrullinated peptide; DAS, Disease Activity Score; MHAQ, Modified Health Assessment Questionnaire; NSAID, non-steroidal anti-inflammatory drug; TNF-a, tumor necrosis factor-alpha; ESR, erythrocyte sedimentation rate; $\mathrm{CRP}, \mathrm{C}$-reactive protein

Zhang and colleagues, 43.2 and 32\%, respectively [31,32] to low prevalence as in Zeytin and colleagues which was $11.5 \%$ [33]. This discrepancy in prevalence was attributed to the differences in the sample size and patient selection.

White matter hyper-intensities as MR imaging feature in migraine patients commonly resemble the white matter lesions observed in inflammatory disorders, such as multiple sclerosis and representing a challenge for diagnosis. However, presence of central vein sign and more than three periventricular lesions is specific for multiple sclerosis $[34,35]$.
In the current study, the prevalence of brain MRI white matter hyper-intensities in RA patient with migraine was $54.8 \%$. This prevalence was higher than that in Le Pira and colleagues and Zhang and colleague. These differences may be explained by the added inflammatory effect of both migraine and rheumatoid disorders on brain micro-vasculature and ischemic brain injuries [31, 32].

In this study, brain MRI was performed for migraine group and exhibited that white matter hyper-intensities (WMHs) were significantly higher in migraine patients with aura $(41.2 \%)$ than migraine patients 
Table 4 Comparisons of quantitative data between migraine cases according to MRI findings

\begin{tabular}{|c|c|c|c|c|}
\hline $\begin{array}{l}\text { Quantitative data } \\
N(\%)\end{array}$ & $\begin{array}{l}\text { Normal brain MRI } \\
28(45.2 \%)\end{array}$ & $\begin{array}{l}\text { Abnormal brain MRI } \\
34(54.8 \%)\end{array}$ & $Z$ value & $P$ value \\
\hline Age (years) & $38.5(34.3-46)$ & 45(38.3-49.8) & 2.125 & 0.034 \\
\hline Rheumatoid disease duration (years) & $6.5(4-8.3)$ & $9.5(5.5-11)$ & 2.431 & 0.015 \\
\hline ESR & $56.5(49-68)$ & $70(55-81.3)$ & 2.288 & 0.022 \\
\hline CRP & $41.5(35-50)$ & $45(38-55)$ & 0.823 & 0.411 \\
\hline Disease Activity Score (DAS-28) & $3.5(2.9-4)$ & $4(3.2-4.2)$ & 1.492 & 0.136 \\
\hline MHAQ & $1.4(1.0-1.9)$ & $1.5(1.0-2.0)$ & 0.666 & 0.505 \\
\hline Migraine duration (years) & $4(2.8-6)$ & $8(4-16)$ & 2.892 & 0.004 \\
\hline
\end{tabular}

Bod values indicate $P$ value is significant (less than 0.05 )

Data are median (25th percentile-75th percentile), test of significance is Mann-Whitney U-test

$P$ value significant $<0.05$, MRI, magnetic resonance imaging; DAS, Disease Activity Score; MHAQ, Modified Health Assessment Questionnaire; ESR, erythrocyte sedimentation rate; CRP, C-reactive protein

without aura $(17.9 \%)$ with statistically significant $P$ value $(p<0.047)$. This was concordant with Rossato and colleagues who found that there was higher prevalence of WMHs in migraine patient with aura due to ischemic brain injury resulted from alteration of cerebral blood flow with subsequent high or low cerebral perfusion which is modulated by cortical spreading depression occurring in aura patients [36].

In the current study, abnormal brain MRI in the form of WMHs were significantly higher in older patients with mean age of about 45 years and in patients with longer migraine duration and with longer rheumatoid duration with statistically significant $P$ value $(p<0.034, P<0.004$ and $P<0.015$, respectively). These were in agreement with $\mathrm{Negm}$ and colleagues and Toghae and colleagues who found that WMHs in migraine patients were directly proportional to patient's age and disease duration [37, 38]. On the other hand, the current study results did not match with the studies of Trauninger and colleagues and Gomez-Beldarrain and colleagues, as they concluded that there was no significant relation regarding patient's age and duration of the disease and they concluded that there was a remission of migraine episodes with aging of the patient $[39,40]$.

In this study, abnormal brain MRI in the form of WMHs was significantly higher in patients with elevated ESR with statistically significant $P$ value $(P<0.22)$, otherwise in this study there was no statistically significant difference regarding CRP, DAS-28 or MHAQ in development of WMHs.

Limitations of the study were small patients sample size, absence of psychiatric assessment and absence of control group study.

\section{Conclusion}

Migraine is highly frequent in rheumatoid arthritis patients, especially migraine with aura. The presence of rheumatoid activity, fibromyalgia and secondary Sjogren's syndrome, elevated ESR and CRP are associated with functional losses in rheumatoid arthritis patients with migraine, especially migraine with aura. MR imaging of brain is a mandatory tool for detection of WMHs in RA patients with migraine, especially in presence of aura.

\section{Abbreviations}

RA: Rheumatoid arthritis; TNF-a: Tumor necrosis factor-alpha; CNS: Central nervous system; MHAQ: Modified Health Assessment Questionnaire; DAS: Disease Activity Score; CBC: Complete blood count; ESR: Erythrocyte sedimentation rate; CRP: C-reactive protein; RF: Rheumatoid factor; Anti-CCP: Anticyclic citrullinated peptide; ICHD-III: International Classification of Headache Disorders -III beta; NRS: Numerical Rating Scale; EM: Episodic migraine; CM: Chronic migraine; MRI: Magnetic resonance imaging; NSAID: Non-steroidal anti-inflammatory drug; WMHs: White matter hyper-intensities.

\section{Acknowledgements}

The authors are grateful to all patients for their willingness to participate in this study.

\section{Authors' contributions}

HAE, CAZ, MRM and AAAE carried out the work. HAE and MRM design the protocol, HAE collected scientific data and share in statistical analysis, AAAE and CAZ shared for collecting the scientific data, did the statistical analysis and were responsible for writing the initial draft of the manuscript, CAZ interpreted the radiology of all patients. All authors read and approved the final manuscript.

\section{Funding}

There is no source of funding for the research.

\section{Availability of data and materials}

The data supporting the results of this article are included within the article. 


\section{Declarations}

\section{Ethics approval and consent to participate}

The authors obtained permission to conduct this study that was approved from Research Ethical Committee (REC) for Human and Animal Research at Faculty of Medicine, Helwan University Serial: 84- 2020 (A). All patients gave written agreement. The procedures followed were in accordance with our protocol. We recruited 220 patients from Outpatient clinic of Rheumatology Department, Helwan University Hospital.

\section{Consent for publication}

Not applicable.

\section{Competing interests}

The authors declare that they have no competing interests.

\section{Author details}

${ }^{1}$ Faculty of Medicine, Helwan University, Cairo, Egypt. ${ }^{2}$ Port Said University, Port fuad, Egypt. ${ }^{3}$ Faculty of Medicine, Ain Shams University, Cairo, Egypt.

Received: 17 June 2021 Accepted: 25 October 2021

Published online: 04 November 2021

\section{References}

1. Collaborators GBDH Global. regional, and national burden of migraine and tension-type headache, 1990-2016: a systematic analysis for the global burden of disease study 2016. Lancet Neurol. 2018;17:954-76.

2. Woldeamanuel YW, Cowan RP. Migraine affects 1 in 10 people worldwide featuring recent rise: a systematic review and meta-analysis of community-based studies involving 6 million participants. J Neurol Sci. 2017;372:307-15.

3. Cavestro C, Ferrero M. Migraine in systemic autoimmune diseases. Endocr Metab Immune Disord Drug Targets. 2018;18(2):124-34.

4. Abou Elmaaty AA, Flifel ME, Belal T, Zarad CA. Migraine and tension headache comorbidity with hypothyroidism in Egypt. Egypt J Neurol Psychiatry Neurosurg. 2020;56:78.

5. Buse DC, Reed ML, Fanning KM, Bostic R, Dodick DW, Schwedt TJ, et al. Comorbid and co-occurring conditions in migraine and associated risk of increasing headache pain intensity and headache frequency: results of the migraine in America symptoms and treatment (MAST) study. J Headache Pain. 2020:21:23.

6. Boer AC, Boonen A, van Mil AHM. Is anti-citrullinated protein antibodypositive rheumatoid arthritis still a more severe disease than anti-citrullinated protein antibody-negative rheumatoid arthritis? A longitudinal cohort study in rheumatoid arthritis patients diagnosed from 2000 onward. Arthritis Care Res. 2018;70(7):987-96.

7. Giles JT. Extra-articular manifestations and comorbidity in rheumatoid arthritis: potential impact of pre-rheumatoid arthritis prevention. Clin Ther. 2019;41(7):1246-55.

8. Le H, Tfelt-Hansen P, Russell MB, Skytthe A, Kyvik KO, Olesen J. Co-morbidity of migraine with somatic disease in a large population-based study. Cephalalgia. 2011;31:43-64

9. Altamura C, Corbelli I, de Tommaso M, Lorenzo CD, Lorenzo GD, Renzo AD. Pathophysiological bases of comorbidity in migraine. Front Hum Neurosci. 2021. https://doi.org/10.3389/fnhum.2021.640574.

10. Mathieu S, Couderc M, Pereira B, Dubost JJ, Malochet-Guinamand S, Tournadre $A$, et al. Prevalence of migraine and neuropathic pain in rheumatic diseases. J Clin Med. 2020;9(6):1890.

11. Kumar $\mathrm{N}, \mathrm{Abboud} \mathrm{H}$. latrogenic CNS demyelination in the era of modern biologics. Mult Scler. 2019;25:1079-85.

12. Wolfe F. Which HAQ is best? A comparison of the HAQ, MHAQ and RA-HAQ, a difficult 8 item HAQ (DHAQ), and a rescored 20 item 'HAQ (HAQ20): analyses in 2,491 rheumatoid arthritis patients following leflunomide initiation. J Rheumatol. 2001;28:982-9.

13. Prevoo ML, Vant-Hof MA, Kuper HH, van Leeuwen MA, van de Putte $L B$, van Riel PL. Modified disease activity score that include twentyeight-joint counts. Development and validation in a prospective longitudinal study of patients with rheumatoid arthritis. Arthritis Rheum. 1995:38:44-8.

14. Mourad D, Hajj A, Hallit S, Ghossoub M, Khabbaz LR. Validation of the Arabic version of the migraine disability assessment scale among Lebanese patients with migraine. J Oral Facial Pain Headache. 2019;33(1):47-53.

15. Headache Classification Committee of the International Headache Society(IHS) The International Classification of Headache Disorders, 3rd edn. Cephalalgia. 2018;38(1):1-211.

16. Olesen J, Bousser MG, Diener HC, Dodick D, First M, Goadsby PJ, et al. New appendix criteria open for a broader concept of chronic migraine. Cephalalgia. 2006;26:742-6.

17. Dijkers M. Comparing quantification of pain severity by verbal rating and numeric rating scales. J Spinal Cord Med. 2010;33:232-42.

18. Tabachnick BG, Fidell LS. Using multivariate statistics. 6th ed. Harlow: Pearson; 2014.

19. Daniel WW, Cross CL. Biostatistics: a Foundation for Analysis in the Health Sciences, 10th edn. New York: Wiley. 2013; 6: 161-213.

20. Wang YC, Huang YP, Wang MT, Wang HI, Pan SL. Increased risk of rheumatoid arthritis in patients with migraine: a population based, propensity score-matched cohort study. Rheumatol Int. 2017:37:273-327.

21. Zeller J, Weissbarth E, Baruth B, Mielke H, Deicher H. Serotonin content of platelets in inflammatory rheumatic diseases. Arthritis Rheum. 1983;26:532-40.

22. Cloëz-Tayarani I, Petit-Bertron AF, Venters HD, Cavaillon JM. Differential effect of serotonin on cytokine production in lipopolysaccharidestimulated human peripheral blood mononuclear cells: involvement of 5-hydroxytryptamine2A receptors. Int Immunol. 2003;15:233-40.

23. Sacre S, Medghalchi M, Gregory B, Brennan F, Williams R. Fluoxetine and citalopram exhibit potent anti-inflammatory activity in human and murine models of rheumatoid arthritis and inhibit toll-like receptors. Arthritis Rheum. 2010;62:683-93.

24. Namas R, Joshi A, Ali Z, Al Saleh J, Abuzakouk M. Demographic and clinical patterns of rheumatoid arthritis in an emirati cohort from United Arab Emirates. Int J Rheumatol. 2019. https://doi.org/10.1155/2019/3057578.

25. Yen JH, Chen JR, Tsai WJ, Liu HW. Correlation of tumor necrosis factor alpha levels with disease activity of rheumatoid arthritis. Zhonghua Minguo wei sheng wu ji mian yi xue za zhi Chin J Microbiol Immunol. 1992;25(4):232-43.

26. Akdag UZ, Kurt S, Karaer UH. The relationship with restless legs syndrome fibromyalgia, and depressive symptoms in migraine patients. Neurol Sci. 2018;39(8):1409-14

27. Pal B, Gibson C, Passmore J, Griffiths ID, Dick WC. A study of headaches and migraine in Sjögren's syndrome and other rheumatic disorders. Ann Rheum Dis. 1989;48(4):312-6.

28. Gökçay F, Öder G, Çelebisoy N, Gökçay A, Sirin H, Kabasakal Y. Headache in primary Sjögren's syndrome: a prevalence study. Acta Neurol Scand. 2008;118:189-92.

29. Morreale M, Marchione P, Giacomini P, Pontecorvo S, Marianetti M, Vento $C$, et al. Neurological involvement in primary Sjögren syndrome: a focus on central nervous system. PLoS ONE. 2014;9:e84605.

30. Tjensvoll AB, Harboe E, Gøransson LG, Beyer MK, Greve OJ, Kvaløy JT, et al. Headache in primary Sjøgren's syndrome: a population-based retrospective cohort study. Eur J Neurol. 2013;20:558-63.

31. Le Pira F, Reggio E, Quattrocchi G, Sanfilippo C, Maci T, Cavallaro T, et al. Executive dysfunctions in migraine with and without aura: what is the role of white matter lesions? Headache. 2013;54(1):125-30.

32. Zhang Q, Datta R, Detre J, Cucchiara B. White matter lesion burden in migraine with aura may be associated with reduced cerebral blood flow. Cephalalgia. 2016;37(6):517-22.

33. Zeytin A, Tok S, Dogan N. The frequency and localization of white matter lesions in migraine patients with or without aura in Anatolia. Turkey Int J Acad Res. 2014;6(2):37-9.

34. Lapucci C, Saitta L, Bommarito G, Sormani MP, Pardini M, Bonzano L, et al. How much do periventricular lesions assist in distinguishing migraine with aura from CIS? Neurology. 2019;92:1-6.

35. Sinnecker T, Clarke MA, Meier D, Enzinger C, Calabrese M, De Stefano $\mathrm{N}$, et al. Evaluation of the central vein sign as a diagnostic imaging biomarker in multiple sclerosis. JAMA Neurol. 2019;76:1446-56.

36. Rossato G, Adami A, Thijs V, Cerini R, Pozzi-Mucelli R, Mazzucco S, et al. Cerebral distribution of white matter lesions in migraine with aura patients. Cephalalgia. 2010;30(7):855-9. 
37. Negm M, Housseini AM, Abdelfatah M, Asran A. Relation between migraine pattern and white matter hyperintensities in brain magnetic resonance imaging. Egypt J Neurol Psychiatry Neurosurg. 2018;54:24.

38. Toghae M, Rahimian E, Abdollahi M, Shoar S, Naderan M. The prevalence of magnetic resonance imaging Hyperintensity in migraine patients and its association with migraine headache characteristics and cardiovascular risk factors. Oman Med J. 2015;30(3):203-7.

39. Trauninger A, Leél-Őssy E, Kamson D, Pótó L, Aradi M, Kövér F, et al. Risk factors of migraine related brain white matter hyperintensities: an investigation of 186 patients. J Headache Pain. 2011;12(1):97-103.
40. Gomez-Beldarrain M, Oroz I, Zapirain B, Ruanova B, Fernandez Y, Cabrera $A$, et al. Right frontoinsular white matter tracts link cognitive reserve and pain in migraine patients. J Headache Pain. 2016;17(1):4.

\section{Publisher's Note}

Springer Nature remains neutral with regard to jurisdictional claims in published maps and institutional affiliations.

\section{Submit your manuscript to a SpringerOpen ${ }^{\circ}$ journal and benefit from:}

- Convenient online submission

- Rigorous peer review

- Open access: articles freely available online

- High visibility within the field

- Retaining the copyright to your article

Submit your next manuscript at $\boldsymbol{\nabla}$ springeropen.com 